\title{
Increased serum level of soluble interleukin- 2 receptor is associated with a worse response of metastatic clear cell renal cell carcinoma to interferon alpha and sequential VEGF-targeting therapy
}

\author{
Akinori Nukui ${ }^{\dagger}$, Akinori Masuda, Hideyuki Abe, Kyoko Arai, Ken-Ichiro Yoshida and Takao Kamai ${ }^{*}$
}

\begin{abstract}
Background: Renal cell carcinoma (RCC) is a tumor with immunogenic properties. Soluble interleukin-2 receptor (sIL-2R) has a role in T cell activation and may be important for immune regulation in various conditions, including infections, transplantation rejection, autoimmune inflammatory states, and cancer. We investigated the prognostic value of the serum sIL-2R level in patients with metastatic RCC receiving IFN-alpha and vascular endothelial growth factor (VEGF)-targeting therapy.
\end{abstract}

Methods: We monitored the serum level of sIL-2R over time and examined phosphorylated Akt expression by the primary tumor in 47 patients with metastatic clear cell RCC (cCRCC) undergoing cytoreductive nephrectomy followed by first-line adjuvant therapy with IFN-alpha plus sequential VEGF-targeting therapy as second- or third-line adjuvant therapy.

Results: A preoperative increase of the serum level of sIL-2R was correlated with a higher preoperative serum level of programmed cell death 1 (PD-1)-ligand 1 (PD-L1), increased expression of phosphorylated Akt by the primary tumor, and a worse response to IFN-alpha/sequential VEGF-targeting therapy, as well as being an independent prognostic factor for a shorter overall survival time by multivariate analysis. Over time, the serum sIL-2R level largely reflected the tumor response to therapy.

Conclusions: Monitoring the serum level of sIL-2R may help to predict the biological behavior of $c C R C C$, its response to IFN-alpha/sequential VEGF-targeting therapy, and the prognosis.

Keywords: Soluble interleulin-2 receptor, Renal cell carcinoma, Interferon, Sorafenib, Axitinib

\section{Background}

The interactions between malignancies and the immune system of the host are extremely complex. Although the immune system theoretically suppresses tumor development and/or promotes tumor regression, it is currently accepted that it can also stimulate tumor growth. These opposing actions of the immune system have been summarized as cancer immunoediting (the three E's: elimination,

\footnotetext{
*Correspondence: kamait@dokkyomed.ac.jp

${ }^{\dagger}$ Equal contributors

Department of Urology, Dokkyo Medical University, 880 Kitakobayashi Mibu, Tochigi 321-0293, Japan
}

equilibrium, and escape) [1], and one of the "hallmarks of cancer" is the ability to evade host immunity [2]. Suppression of tumor development requires the generation and activation of tumor antigen-specific $\mathrm{T}$ cells, so activating the immune system to treat cancer via stimulation of $\mathrm{T}$ cells has long been an objective of studies on antitumor immunity. Multiple co-stimulatory receptors and negative regulators (or co-inhibitory receptors) interact to regulate the activation and proliferation of T cells, as well as the gain or loss of $\mathrm{T}$ cell effector function [3, 4].

Clear cell renal cell carcinoma (ccRCC) has the typical features of an immunogenic tumor, including numerous 
tumor-infiltrating $\mathrm{T}$ lymphocytes (TILs) and cytotoxic $\mathrm{T}$ cells, which identify and selectively destroy tumor cells, as well as circulating tumor-specific T cells $[5,6]$. CD4positive $(\mathrm{CD} 4+\mathrm{CD} 25+$ Foxp3+) regulatory $\mathrm{T}$ cells (Tregs) play an essential role in immunosuppression and self-tolerance of tumor antigens in patients with cancer or tolerance of microbial antigens in patients with chronic infection [7]. It was reported that patients with metastatic RCC show an increase of CD4+ Tregs, while high-dose IL-2 significantly decreases CD4+ Tregs in patients with an objective response to this cytokine [8].

IL-2 is important for the growth and differentiation of both effector $\mathrm{T}$ (Teff) cells and CD4+ Tregs, thus promoting either immunostimulation or immunosuppression, and it not only has a critical role in protective immunity but also in peripheral immune tolerance mediated by CD4 + Tregs [9-11]. IL-2 must bind with the IL-2 receptor (IL-2R) on target cells to mediate these various actions. There are three subunits of the IL-2R: IL-2R alpha (CD25), IL-2R beta (CD122), and IL-2R gamma (CD132) [9-11]. T cells constitutively express IL-2R beta and IL-2R gamma, while expression of IL-2R alpha increases with $\mathrm{T}$ cell activation. Thus, IL-2R alpha can serve as a phenotypic marker for CD4+ Tregs [7]. IL-2R alpha is released by $\mathrm{T}$ cells as a soluble form (soluble interleukin- 2 receptor: sIL-2R), and elevation of the sIL-2R level is detected in patients with infectious diseases, transplantation rejection, autoimmune inflammation, and cancer [9-11]. Thus, it seems that sIL-2R release promotes T cell activation and is important for immune regulation in various conditions.

IL-2R signaling regulates tolerance and immunity by inducing the transcription of target genes (such as CD25) via various signaling pathways, such as the Janus kinase (JAK)-signal transducer and activator of transcription (STAT) pathway; the phosphatidylinositol 3'kinase (PI3K), serine/threonine kinase Akt, and mammalian target of rapamycin (mTOR) pathway; and the mitogen-activated protein kinase (MAPK) pathway [9-11]. Among these pathways, there is marked activation of the PI3K/Akt/ mTOR pathway in RCC [12]. Through cancer immunoediting, CD4+ Treg cells and programmed cell death 1 (PD1)-ligand 1 (PD-L1) play an important role in promoting the escape phase of tumor growth in an immunosuppressive tumor microenvironment [1], while interaction between PD-1 and PD-L1 may be involved in the production of CD4+ Tregs [4]. In addition, aerobic glycolysis in tumor cells promotes depletion of extracellular glucose and leads to dysfunction of TILs, while expression of PD-L1 in tumor cells leads to constitutive activation of the Akt/ mTOR pathway $[13,14]$. Thus, sIL-2R could potentially be a biomarker for prediction of resistance and selection of therapy, but its role in human RCC has not been elucidated. Accordingly, we investigated serum sIL-2R, serum PD-L1, and phosphorylated Akt expression by the primary tumor in patients with metastatic ccRCC undergoing cytoreductive nephrectomy followed by IFN-alpha and sequential VEGF-targeting therapy. Our findings provide some insight into the clinical utility and biological significance of sIL-2R in ccRCC patients.

\section{Methods \\ Patients}

This was a retrospective study performed in 47 patients (32 men and 15 women) with histopathologically diagnosed metastatic ccRCC who underwent cytoreductive nephrectomy at our center between June 2007 and June 2014. Patients received cytoreductive nephrectomy before undergoing any other therapy. For staging of the tumor, all patients underwent preoperative CT and/or MRI. Postoperative follow-up ranged from 3 to 100 months, with a median of 31 months. Metastatic disease was evaluated by $\mathrm{CT}$ and/or MRI every 2 to 4 months. This study was conducted in accordance with the Declaration of Helsinki and was approved by the ethical review board of Dokkyo Medical University Hospital. Each patient signed a consent form that was approved by our institutional Committee on Human Rights in Research.

Enrollment criteria for this study were as follows: (1) age $\geq 18$ years; (2) detection of metastatic disease at the time of cytoreductive nephrectomy for ccRCC; (3) firstline IFN-alpha therapy that was discontinued for medical reasons (e.g., progressive disease, stable disease, or intolerable adverse effects); (4) IFN-alpha plus low-dose sorafenib as second-line therapy with/without axitinib as third-line therapy (patients refractory to IFN-alpha plus sorafenib); and (5) available medical records for the entire period from the start of first-line therapy until final follow-up/death.

After cytoreductive nephrectomy, all 47 patients received adjuvant immunotherapy with IFN-alpha to treat their extra-renal disease. First-line therapy was provided with natural human IFN-alpha (3, 5, or 6 million units administered intravenously or intramuscularly 2-3 times weekly). Patients with refractory tumors (progressive disease: PD) received second-line therapy, which was IFN-alpha (3, 5, or 6 million units intravenously or intramuscularly 2-3 times weekly) combined with low-dose sorafenib $(400 \mathrm{mg} /$ day $=50 \%$ of the recommended starting dose) [15]. Since the recommended dose intensity of IFN-alpha and anti-VEGF agents is lower in Japan than in the USA or EU due to the smaller physique of Japanese patients, we administered a low dose of sorafenib to reduce toxicity and combined it with IFN-alpha to increase the antitumor activity $[15,16]$. Some patients who showed a poor response to second-line therapy with IFN-alpha plus sorafenib subsequently received thirdline therapy with axitinib (at the recommended starting dose of $10 \mathrm{mg} /$ day). The attending physicians assessed 
tumor progression on the basis of imaging findings (enlargement of existing lesions or detection of new lesions), deterioration of the performance status, and exacerbation of symptoms such as cancer pain, fever, or weight loss. Dose reduction of IFN-alpha, sorafenib, and axitinib was performed for grade 3/4 toxicity. The response to treatment was assessed according to RECIST criteria [17]. Serum levels of sIL-2R (normal range: 135.0$483.0 \mathrm{U} / \mathrm{ml}$ ) were measured every 1 to 3 months by LSI Medience Corporation (Tokyo, Japan), and the preoperative serum level of soluble PD-L1 was measured by using human PD-L1 (CSB-E13644h, Cusabio Biotech, Wuhan, China). The final follow-up date was determined by reviewing the medical records in October 2015.

\section{Western blotting}

Samples from the resected primary tumors were subjected to western blotting, as reported previously $[15,18]$. To compensate for variation in the expression of phosphorylated Akt (Ser-473) (pAkt(Ser-473)), tumor tissue samples and non-tumor tissue samples from the same patient were compared. The following antibodies were employed: a rabbit anti-human antibody targeting pAkt (Ser-473) (Cell Signaling Technology, Inc; PhosphoPlus Akt (Ser-473) Antibody Kit; \# 9270, Danvers, MA) and a beta-actin antibody (Millipore; \# 1501R Bedford, MA).

\section{Statistical analysis}

The Mann-Whitney $U$ test was performed to compare two groups, while the Kruskal-Wallis test was employed for comparisons among at least three groups. Spearman's rank correlation coefficient analysis was performed to assess the relationships between variables of interest. Cause-specific survival curves were created by the Kaplan-Meier method and differences between the curves were assessed with the log-rank test. The impact on survival of the preoperative sIL-2R level, preoperative PD-L1, pAkt(Ser-473), histological grade, pT stage, pN stage, and microscopic vascular invasion was investigated by univariate and multivariate Cox proportional hazards analysis. In all analyses, $P<0.05$ indicated statistical significance. Analyses were done with commercial software [18].

\section{Results}

The clinical characteristics and outcomes of the patients are summarized in Table 1.

The preoperative serum sIL-2R level ranged from 114.2 to $2200.9 \mathrm{U} / \mathrm{ml}($ mean \pm S.D. $=601.5 \pm 503.8 \mathrm{U} /$ $\mathrm{ml})$. None of the patients had inflammatory and/or autoimmune diseases, so preoperative sIL-2R levels exceeding the median value $(498.8 \mathrm{U} / \mathrm{ml})$ were not derived from concomitant diseases.
An increase of the preoperative sIL-2R level was detected in patients with poorly differentiated cancer (Fuhrman grade $1 / 2$; mean \pm S.D. $=322.9 \pm 264.6$, Fuhrman grade $3 /$ 4; $778.8 \pm 594.5, P=0.002$ ), local invasion (pT1/2; mean \pm S.D. $=230.7 \pm 111.5, \mathrm{pT} 3 / 4 ; 667.9 \pm 556.9$, $P=0.0146)$, lymph node metastasis $(\mathrm{pN} 0$; mean \pm S.D. $=$ $490.0 \pm 506.7, \mathrm{pN} 1 / 2 ; 834.6 \pm 543.3, P=0.0143)$, and vascular invasion (negative; mean \pm S.D. $=269.0 \pm 217.6$, positive; $673.3 \pm 560.4, P=0.0276$ ).

Among 47 patients who had metastasis when they underwent cytoreductive nephrectomy and received IFN-alpha as first-line adjuvant therapy, six patients showed a complete response (CR), partial response (PR), or stable disease (SD) for $>24$ weeks, while progression occurred in the other 41 patients and they were given IFN-alpha combined with low-dose sorafenib as secondline therapy. When evaluated from the best response, 19 of these 41 patients displayed a good response to IFNalpha plus sorafenib, while the other 22 patients did not respond. Eight of the 19 responders eventually became resistant to second-line therapy. Ten of the 22 nonresponders subsequently received best supportive care. Among the 20 patients $(12 / 22$ non-responders and 8/19 responders to second-line therapy) who received axitinib as third-line therapy, nine patients (4/12 non-responders and $5 / 8$ responders to second-line therapy) showed a good response, while the remaining 11 patients were non-responders.

Preoperative sIL-2R level and response of metastatic cCRCC A lower preoperative serum sIL-2R level showed a correlation with a good response (complete response, partial response, or stable disease for $>24$ weeks) to either IFN-alpha monotherapy, IFN-alpha plus sorafenib, or axitinib (Table 2). When the patients displaying a good response to IFN-alpha $(n=6)$, IFN-alpha plus sorafenib $(n=19)$, or axitinib $(4 / 12$ non-responders to second-line therapy) were combined in a good response group $(n=29)$, the preoperative serum sIL-2R level was lower in this group compared with the group showing a poor response to any of these agents (i.e., stable disease for $<24$ weeks or progressive disease) $(P=0.0046$, Table 2).

Analysis of the time course of serum sIL-2R levels revealed that they largely paralleled the response to therapy (Fig. 1). For example, serum sIL-2R began to increase if a patient had a poor response to therapy and continued to increase thereafter (Fig. 2a), sIL-2R remained stable (even if it was high) or decreased gradually in patients with relatively long-term stable disease (Fig. 2b), sIL-2R was stable within the normal range or decreased toward normal in patients with a good response to any of the agents (Fig. 2c), or sIL-2R remained high and continued to rise further in patients responding poorly to any agent (Fig. 2d). 
Table 1 Background of 47 metastatic clear cell RCCs

\begin{tabular}{|c|c|c|c|c|c|c|}
\hline & & 1st line & 2nd line $(n=41)$ & & 3rd line $(n=20)$ & \\
\hline & & IFN-alpha & IFN-alpha + sorafe & & Axitinib & \\
\hline & & $\begin{array}{l}\text { CR/PR/SD }>24 w \\
(n=6)\end{array}$ & $\begin{array}{l}\text { CR/PR/SD }>24 \mathrm{~W} \\
(n=19)\end{array}$ & $\begin{array}{l}\mathrm{SD}<24 \mathrm{~W} / \mathrm{PD} \\
(n=22)\end{array}$ & $\begin{array}{l}\mathrm{CR} / \mathrm{PR} / \mathrm{SD}>24 \mathrm{~W} \\
(n=9)\end{array}$ & $\begin{array}{l}\mathrm{SD}<24 \mathrm{w} / \mathrm{PD} \\
(n=11)\end{array}$ \\
\hline Sex (Male / Female) & $32 / 15$ & & & & & \\
\hline Years (median) & $39-78(65)$ & & & & & \\
\hline ECOG PS ${ }^{a}(0 / 1 / 2)$ & $29 / 14 / 4$ & $6 / 0 / 0$ & $13 / 6 / 0$ & $10 / 8 / 4$ & $4 / 5 / 0$ & $2 / 8 / 1$ \\
\hline MSKCC (Fav / Int / Poor) & $24 / 15 / 8$ & $6 / 0 / 0$ & $9 / 7 / 0$ & $9 / 8 / 8$ & $2 / 7 / 0$ & $1 / 8 / 2$ \\
\hline $\begin{array}{l}\text { Duration of IFN-alpha } \\
\text { (mean: months) }\end{array}$ & & $7-46(15.9)$ & & & & \\
\hline $\begin{array}{l}\text { Duration of pre-IFN-alpha }{ }^{a} \\
\text { (mean: months) }\end{array}$ & & & $1-31(7.4)$ & & & \\
\hline $\begin{array}{l}\text { Duration of IFN-alpha + sorafenib } \\
\text { (mean: months) }\end{array}$ & & & $1-81(19.7)$ & & & \\
\hline Duration of axitinib (mean: months) & & & & & $1-37(11.6)$ & \\
\hline Metastatic lesions ${ }^{a}$ (numbers) & & & & & & \\
\hline PUL & 46 & 6 & 18 & 22 & 9 & 11 \\
\hline PLE & 6 & 1 & 2 & 3 & 2 & 2 \\
\hline HEP & 7 & 0 & 3 & 4 & 2 & 2 \\
\hline OSS $^{\mathrm{a}}$ & 11 & 0 & 6 & 5 & 3 & 3 \\
\hline LYM & 12 & 0 & 6 & 6 & 3 & 4 \\
\hline Others & 3 & 0 & 0 & 3 & 0 & 2 \\
\hline
\end{tabular}

ECOG PS ${ }^{\text {a }}$ Eastern Cooperative Oncology Group (ECOG) performance status

MSKCC $^{\mathrm{a}}$ : Memorial Sloen-Kettering Cancer Center, Fav Favorable, Int Intermediated, Poor Poor risk

Duration of IFN-alpha ${ }^{a}$ : Duration of IFN-alpha monotherapy

Duration of pre-IFN-alpha ${ }^{a}$ : Duration of IFN-alpha monotherapy prior tolFN-alpha plus sorafenib

Metastatic lesions ${ }^{\mathrm{a}}$; PUL Lung, PLE Pleura, HEP Liver, OSS Bone, LYM lymph node

OSS $^{\mathrm{a}}$ : Treatment option with Radiation plus Bisphosphonate or Denosmab

Elevated pAkt(Ser-473) expression in the primary tumor was found to show a correlation with a poor response to IFN-alpha and sequential VEGF-targeting therapy $(P=0.0021$, Table 2$)$.

When the relation between the preoperative serum sIL-2R level and pAkt(Ser-473) expression by the primary tumor was investigated, sIL-2R was positively correlated with pAkt(Ser-473) $\left(\mathrm{r}^{2}=0.59, P=0.00003\right.$, Fig. 3a).

Elevation of the preoperative serum level of PL-L1 was also found to be associated with a poor response to IFN-alpha and sequential VEGF-targeting therapy

Table 2 Relationship between molecules and treatment outcome

\begin{tabular}{|c|c|c|c|c|c|c|}
\hline & $S I L-2 R$ & $p$ value & PD-L1 & $p$ value & pAkt (Ser-473) & $p$ value \\
\hline & $(\mathrm{U} / \mathrm{ml})$ & & $(\mathrm{pg} / \mathrm{ml})$ & & & \\
\hline & mean $\pm S . D$ & & mean $\pm S . D$ & & mean $\pm S . D$ & \\
\hline \multicolumn{7}{|l|}{ IFN-alpha group } \\
\hline IFN alone: CR/PR/SD > 24W* $(n=6)$ & $123.9 \pm 43.1$ & 0.01 & $17.3 \pm 13.0$ & 0.02 & $2.67 \pm 0.93$ & 0.01 \\
\hline IFN + Sor: CR/PR/SD > 24w* $(n=19)$ & $331.6 \pm 207.7$ & & $18.6 \pm 15.8$ & & $3.93 \pm 2.09$ & \\
\hline IFN + Sor: SD $<24 \mathrm{~W} / \mathrm{PD}^{*}(n=22)$ & $567.3 \pm 577.6$ & & $60.1 \pm 98.2$ & & $6.42 \pm 2.52$ & \\
\hline Axitinib: CR/PR/SD > 24W* $(n=9)$ & $433.3 \pm 205.2$ & & $27.2 \pm 16.4$ & & $5.95 \pm 2.85$ & \\
\hline Axitinib: SD $<24 \mathrm{~W} / \mathrm{PD}^{*}(n=11)$ & $1050.1 \pm 710.8$ & & $43.8 \pm 24.6$ & & $7.01 \pm 2.57$ & \\
\hline \multicolumn{7}{|l|}{ 1st and/or 2nd and/or 3rd therapy* } \\
\hline $\mathrm{CR} / \mathrm{PR} / \mathrm{SD}>24 \mathrm{~W}^{*}(n=29)$ & $367.1 \pm 242.3$ & 0 & $18.0 \pm 12.9$ & 0 & $3.69 \pm 1.97$ & 0 \\
\hline $\mathrm{SD}<24 \mathrm{~W} / \mathrm{PD}^{*}(n=18)$ & $784.5 \pm 558.4$ & & $41.6 \pm 20.7$ & & $6.67 \pm 2.51$ & \\
\hline
\end{tabular}

$\mathrm{CR} / \mathrm{PR} / \mathrm{SD}>24 \mathrm{w}^{*}$ : complete, partial, or stable with $>24$ weeks response

$\mathrm{SD}<24 \mathrm{w} / \mathrm{PD}^{*}$ : stable disease for $<24$ weeks or progressive disease

1 st and/or 2nd and/or 3rd therapy* : IFN-alpha (1st-line), IFN-alpha + Sorafenib (2nd-line), Axitinib (3rd-line) 


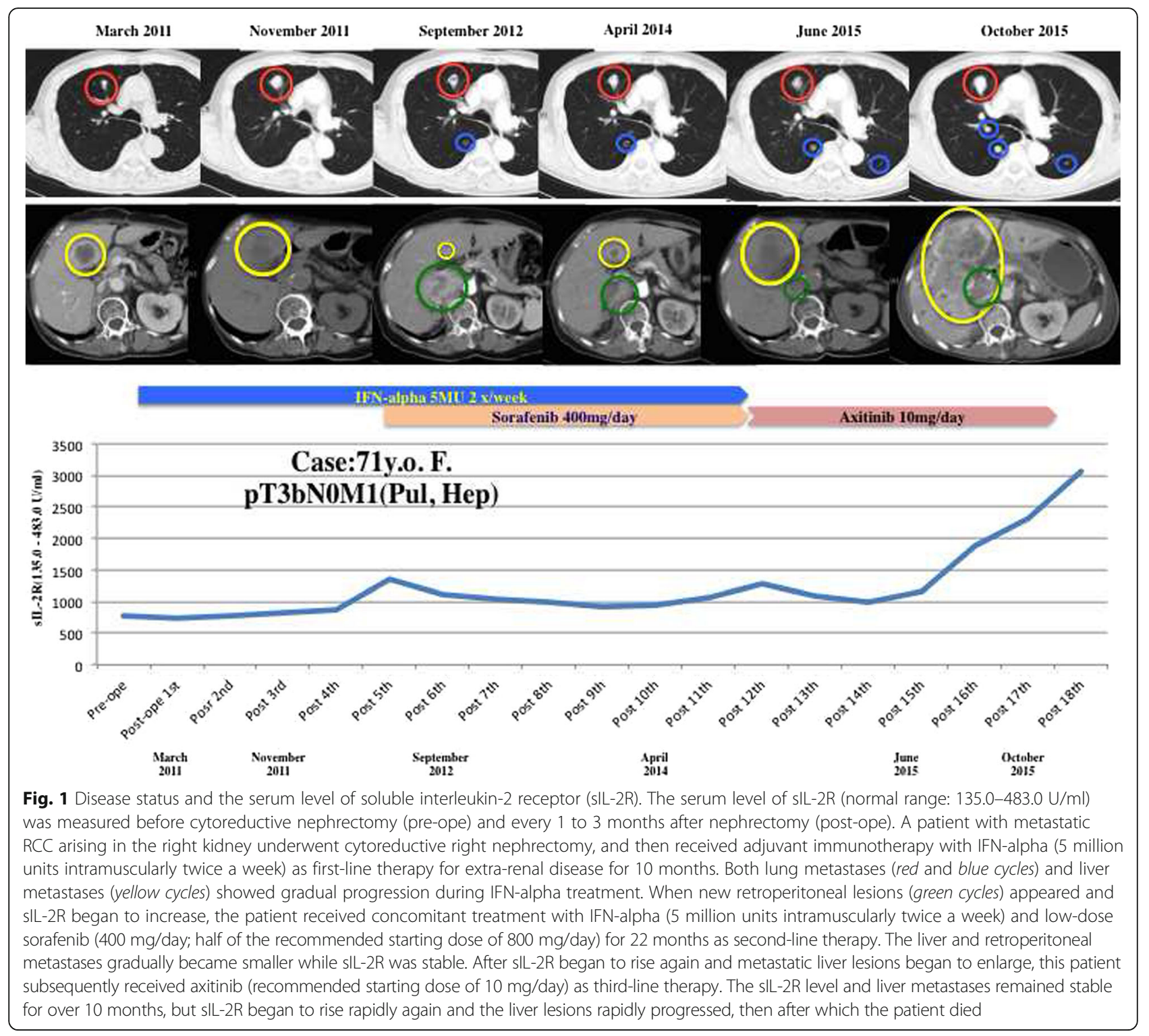

$(P=0.00004$, Table 2$)$, while preoperative sIL-2R and PD-L1 levels demonstrated a positive correlation $\left(r^{2}=0.54, P=0.00009\right.$, Fig. 3b).

Association of the serum sIL-2R level with overall survival The median overall survival time (OS) of all patients after cytoreductive nephrectomy and IFN-alpha therapy was 31.9 months (Fig. 4a).

In patients with a favorable response to either IFNalpha as first-line therapy, IFN-alpha plus low-dose sorafenib as second-line therapy, or axitinib as third-line therapy, median OS was 47.2 months, while median OS was only 11.9 months for patients responding poorly to any agent $(P=0.0033$, Fig. $4 b)$.

When the median preoperative serum level of sIL-2R (498.8 U/ml) was employed as the cut-off value to divide the patients into two groups, a higher sIL-2R level and shorter overall survival were associated according to Kaplan-Meier analysis $(P=0.00007$, Fig. $4 \mathrm{c})$.

A higher serum sIL-2R level (median: 498.8), higher serum PD-L1 level (median: 27.0), higher pAkt(Ser-473) expression (median: 5.63), undifferentiated tumor histology, and regional lymph node metastasis were all associated with shorter overall survival according to Cox univariate analysis, but only sIL-2R and PD-L1 were confirmed to have an impact by multivariate analysis (Table 3 ).

\section{Discussion}

The main findings of the present study were as follows: 1) patients with higher preoperative sIL-2R levels showed a worse response to IFN-alpha and sequential VEGFtargeting therapy, and multivariate analysis demonstrated 


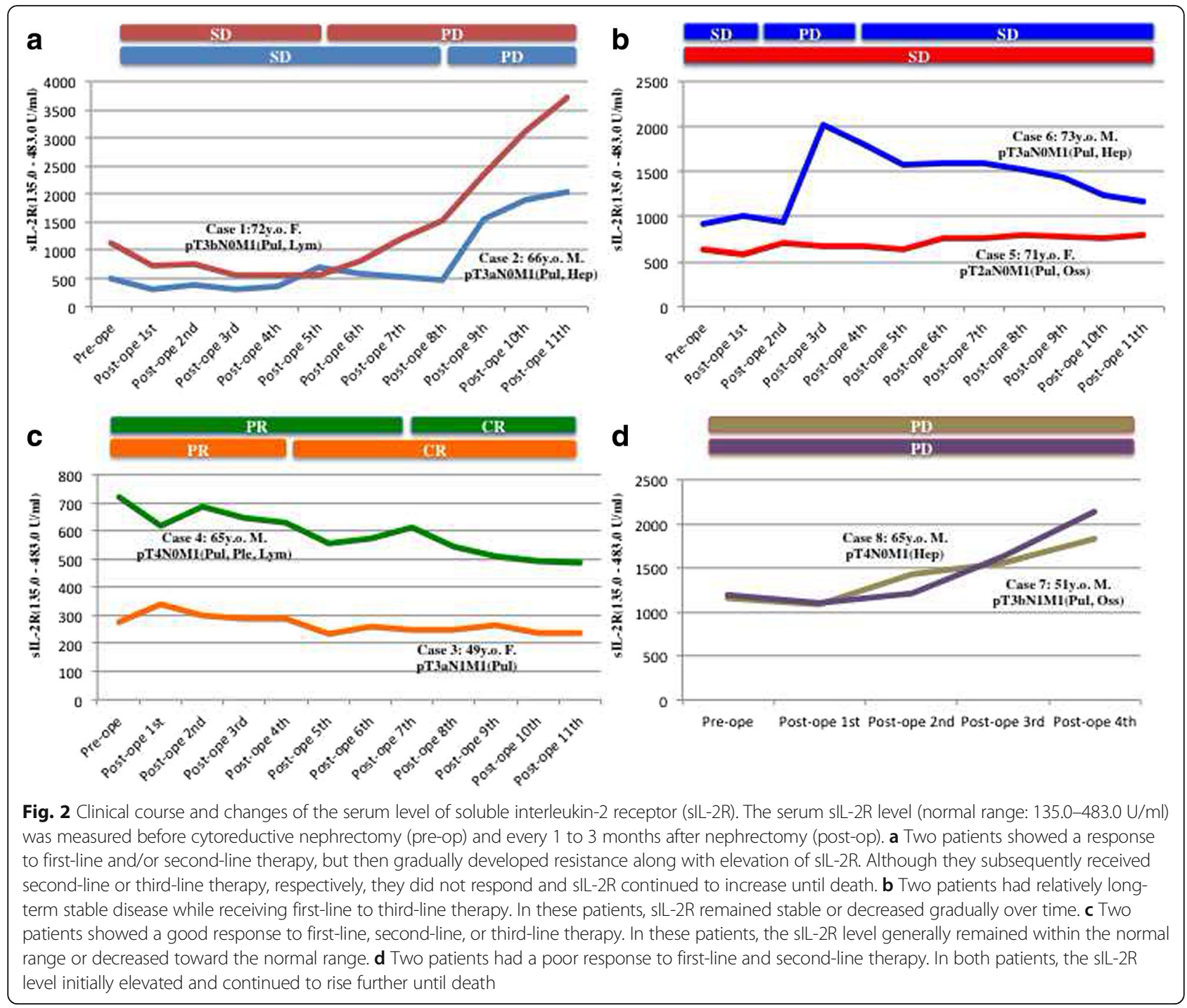

that preoperative elevation of sIL-2R was an independent prognostic factor for shorter overall survival. 2) The serum level of sIL-2R largely paralleled the response to therapy over time. 3) Preoperative serum sIL-2R displayed a positive correlation with preoperative serum soluble PD-L1 and with expression of pAkt(Ser-473) by the primary tumor. Since blood samples are easier to obtain than tissue samples, blood biomarkers are preferable for assessing tumor progression and the response to therapy, as well as for personalized treatment. Our findings suggest that sIL$2 R$ could possibly be employed to assess the biological behavior and progression of ccRCC, as well as to predict the response to IFN-alpha combined with sequential VEGFtargeting therapy.

IL-2R signaling has an important role in tolerance and in the immune response [9-11]. Tregs are a subset of CD4+ $\mathrm{T}$ cells that constitutively express CD25 (alphachain of the IL-2R), and are involved in immunoregulation
[7]. Serum sIL-2R and the number of CD4+ Tregs were reported to display a positive correlation in cancer patients [19]. Tumors express numerous antigens, including self-antigens. Tregs are essential for suppression of $\mathrm{T}$ cell responses to tumor-associated antigens and for maintaining tolerance to self-antigens [7].

IL-2 and IL-2R are involved in immune responses by inducing the PI3K/Akt/mTOR pathway [9-11], and this pathway is highly activated in RCC [12]. Inhibition of Akt blocks transcription of glucose transporter protein-1 (GLUT1) and its translocation to the plasma membrane, where it promotes glucose utilization independently of any proliferative effect [20]. Increased glucose uptake, mainly mediated by GLUT-1, is associated with the increased dependence of tumor cells on glycolysis in the presence of oxygen (Warburg effect), and such reprogramming of cellular metabolism is considered to be a "hallmark of cancer" [2]. RCC demonstrated a shift of metabolism towards 


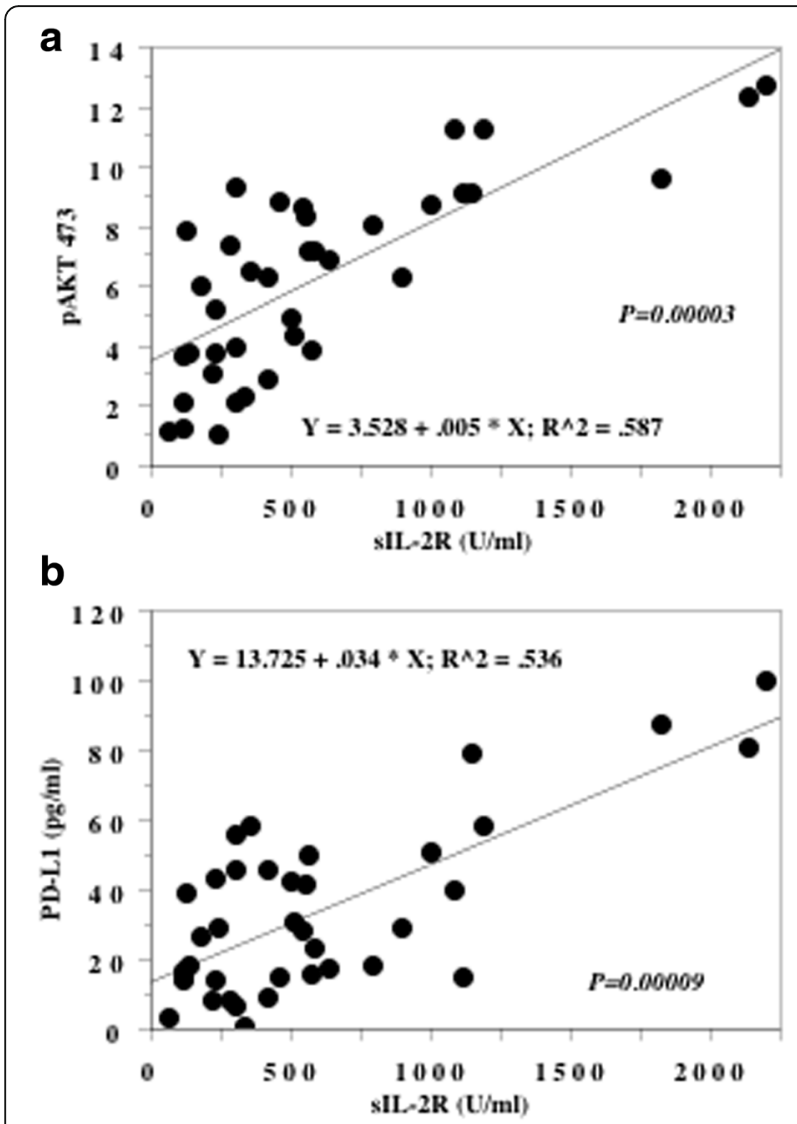

Fig. 3 Spearman rank correlation between sIL-2R and Akt in the primary tumors and PD-L1. Spearman rank correlation between the preoperative serum sIL-2R level and the expression levels of phosphorylated Akt(Ser-473) in the primary tumors (a), and preoperative serum PD-L1 level (b)

aerobic glycolysis due to impaired oxidative phosphorylation [21]. There are two forms of mTOR, mTOR complex 1 (mTORC1) and mTOR complex 2 (mTORC2), which have different intracellular functions. mTORC1-pS6 signaling promotes translation and protein synthesis, while mTORC2-pAkt(Ser-473) signaling influences energy metabolism and cell survival [22], and it has a very important role in RCC [23]. We recently reported that elevated pAkt(Ser-473) expression by the primary tumor shows a correlation with the invasiveness and metastatic potential of RCC, as well as with an unfavorable prognosis [18]. We have also previously demonstrated that higher expression of pAkt(Ser-473) in the primary tumor leads to a worse response of metastases to treatment with IFN-alpha plus lowdose sorafenib [15]. In addition, Jonasch et al. reported that detection of increased pAkt(Ser-473) expression by microarray analysis was related to worse survival after treatment with IFN-alpha plus sorafenib [24]. In the present study, the preoperative serum sIL-2R level was positively correlated with pAkt(Ser-473) expression by the primary tumor, and patients with higher preoperative
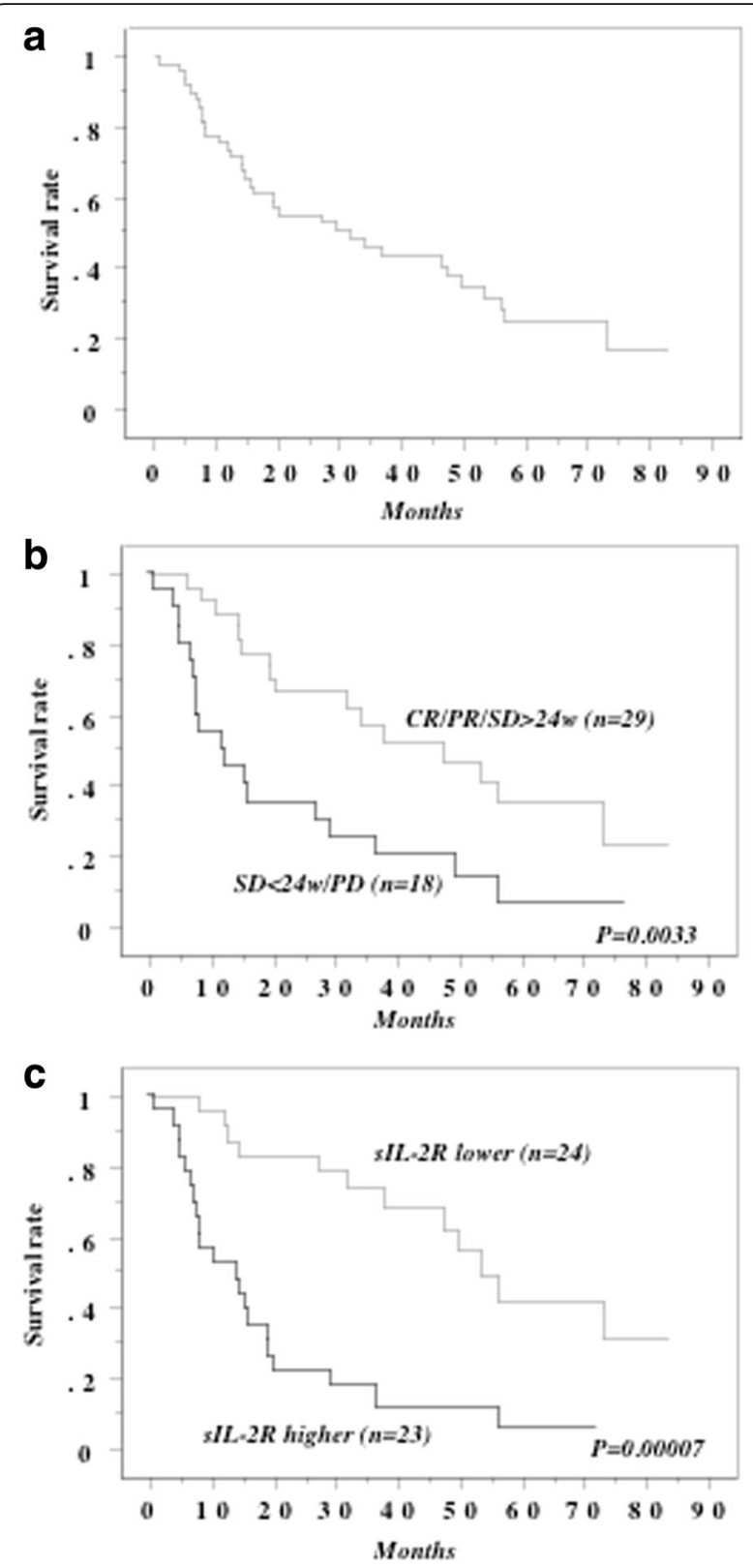

Fig. 4 Overall survival curve in all patients. a Overall survival curve in all patients. b The patients with better response either for IFN-alpha, IFN-alpha plus sorafenib, or axitinib showed longer survival than those with poorer response. c This survival curve is based on the median values of preoperative serum SIL-2R level in all cases. The cases were divided into two groups at this level - high and low value. $P$ value was analyzed by log-rank test

sIL-2R levels and higher pAkt(Ser-473) expression showed a poor response to IFN-alpha with sequential VEGF-targeting therapy. Although we did not investigate the relationship of sIL-2R to pAkt(Ser-473) or the direct role of sIL-2R in tumor progression, our findings suggested that the serum sIL-2R level may reflect the biological aggressiveness of RCC. Accordingly, the role 
Table 3 Cox regression analysis for various potential prognostic factors in overall survival

\begin{tabular}{|c|c|c|c|c|c|c|c|c|}
\hline \multirow[t]{2}{*}{ Variable } & \multirow{2}{*}{$\begin{array}{l}\text { Unfavorable/ } \\
\text { favorable } \\
\text { characteristics }\end{array}$} & \multirow{2}{*}{$\begin{array}{l}\text { No. of } \\
\text { patients }\end{array}$} & \multicolumn{3}{|l|}{ Univariate $(U)$} & \multicolumn{3}{|c|}{ Multivariate (M) } \\
\hline & & & Relative risk & 95\% confidential interval & $P$ value & Relative risk & 95\% confidential interval & $P$ value \\
\hline SIL-2R & high / low & $23 / 24$ & 4.44 & $2.094-9.426$ & 0 & 2.62 & $1.161-6.452$ & 0.0167 \\
\hline PD-L1 & high / low & $23 / 24$ & 3.980 & $1.891-8.378$ & 0 & 3.89 & $1.389-10.898$ & 0.0097 \\
\hline pAkt & high / low & $23 / 24$ & 2.91 & $1.189-7.137$ & 0.02 & 1.59 & $0.651-2.326$ & 0.4539 \\
\hline Grade & $4 / 3 / 2 / 1$ & $4 / 21 / 19 / 3$ & 3.105 & $1.711-5.634$ & 0 & 1.87 & $0.974-3.588$ & 0.06 \\
\hline pT & $4,3 / 2,1$ & $38 / 9$ & 2.04 & $0.766-5.411$ & 0.15 & & & \\
\hline $\mathrm{pN}$ & $2,1 / 0$ & $13 / 34$ & 2.79 & $1.335-5.847$ & 0.01 & 2 & $0.835-4.803$ & 0.12 \\
\hline Vascular invasion & $1 / 0$ & $39 / 8$ & 2.56 & $0.890-7.347$ & 0.08 & & & \\
\hline
\end{tabular}

of sIL-2R in ccRCC should be investigated further in the future.

SIL-2R is generated by proteolytic cleavage and extracellular release of the membrane-bound form of IL-2R alpha [9-11]. sIL-2R release is associated with $\mathrm{T}$ cell activation and seems to be important for regulation of immunity in various settings, including infections, transplantation rejection, autoimmune inflammatory states, and cancer [9-11]. sIL-2R is reported to be produced by tumor cells and sIL$2 \mathrm{R}$ levels are increased in non-Hodgkin's lymphoma, but the reasons why elevation of sIL-2R influences the prognosis are unclear. It has been suggested that sIL-2R suppresses IL-2R signaling and activates Tregs to promote tolerance of malignancy by the host immune system, leading to a poor prognosis [9-11]. Tregs promote immunosuppression and tolerance to tumor antigens in cancer patients, and these cells play the same role for microbial antigens in chronic infection [7]. An increase of circulating Tregs was reported in patients with RCC [25]. IL-2/IL-2R signaling has contradictory immunomodulatory effects, since it not only facilitates proliferation of cytotoxic CD8 T cells that kill cancer cells, but also suppresses the immune response by promoting inhibitory $\mathrm{CD} 4^{+}$Treg cells [9-11]. Accordingly, it can be suggested that the immunosuppressive state arising due to increased generation of Tregs may be associated with or reflected by abnormal elevation of sIL-2R.

Before metastasis occurs, cells originating from the bone marrow are recruited to the lungs, where these cells form clusters that facilitate adherence and proliferation of circulating tumor cells [26]. These marrow-derived cells produce matrix metalloproteinase (MMP)-9, which may promote invasion by cancer cells [27]. Yoshida et al. reported that serum sIL-2R and the number of CD68positive macrophages in the tumor microenvironment were positively correlated, and functional studies performed in lymphoma have shown that MMP-9 is largely produced by tumor-associated macrophages (TAMs) and plays an important role in facilitating sIL-2R production [28]. Myeloid-derived suppressor cells, which phenotypically resemble partially differentiated granulocyte- macrophages and myeloid precursors of the monocytic lineage, are dramatically increased in the circulation of tumor-bearing animals and patients with cancer. Under certain experimental conditions, these progenitor cells undergo differentiation into antigen-presenting cells (APCs), including dendritic cells and macrophages [29]. TAMs are the major inflammatory cell population in tumors and orchestrate various processes, such as the diversion and twisting of adaptive responses, tumor vessel growth, tumor cell proliferation, deposition and remodeling of intercellular matrix, and creation of a metastatic niche with subsequent metastasis, as well as influencing the response to hormones or chemotherapy [30]. MMP-9 is required for production of sIL-2R and TAMs are the main source of MMP-9. Since myeloid-derived suppressor cells, especially TAMs, promote tumor growth by acting in the local tumor microenvironment, it seems likely that TAMs, MMP9, and sIL-2R all play a role in establishing an immunosuppressive environment that facilitates tumor progression. Tumors express a large variety of antigens, which include self-antigens. Tumors are infiltrated by Tregs and myeloid-derived suppressor cells that block local $\mathrm{T}$ cell responses through direct cell-cell contact [7, 29]. Accordingly, the relation of sIL-2R to Tregs and myeloid-derived suppressor cells circulating in the blood or in tumor tissues should be investigated in patients with RCC.

Cancer immunoediting allows an immunologically sculpted tumor to begin to grow progressively in the escape phase until the lesion becomes clinically apparent and establishes an immunosuppressive microenvironment, and immunoediting also promotes tumor growth in which poorly immunogenic and immunoevasive transformed cells are key players along with CD8+ T cells, CD4+ Tregs, and PD-L1 [1]. Both preclinical and clinical studies have demonstrated that suppressing the PD-1/PD-L1 pathway leads to augmentation of antitumor activity, partly by increasing the CD4+ Teff-Treg ratio within tumors [31]. Therefore, targeting $\mathrm{T}$ cells with anti-PD-1/PD-L1 antibodies may possibly overcome the escape mechanisms employed by malignancies and restore the equilibrium of the immune 
system or even facilitate tumor destruction [3, 4]. Recent studies have shown that active glycolysis in tumor cells depletes extracellular glucose and restricts its availability to host cells, leading to impairment of $\mathrm{T}$ cell glycolytic metabolism, while the expression of PD-L1 by tumor cells promotes constitutive activation of the Akt/mTOR pathway and treatment with anti-PD-L1 antibodies attenuates both glycolysis and phosphorylation of Akt [13, 14]. Therefore, our finding that sIL-2R is associated with PD-L1 and pAkt(Ser-473) has implications in relation to tumor biology and host-tumor interactions, suggesting that it may be worthwhile to determine the molecular mechanisms through which sIL-2R, PD-L1, and pAkt act cooperatively or independently in the tumor microenvironment.

This study had several limitations, including its retrospective design, investigation of a relatively small patient population, and a short follow-up period. However, we showed that an elevated preoperative serum level of sIL$2 \mathrm{R}$ was an independent prognostic factor for poor overall survival. The preoperative sIL-2R level was positively correlated with the preoperative serum level of soluble PD-L1 and with expression of pAkt(Ser-473), which has a role in progression of RCC, by the primary tumor. Furthermore, we found that the serum sIL-2R level remained nearly constant when RCC showed a good response to IFN-alpha with sequential VEGF-targeting therapy, while sIL-2R began to increase when resistance to therapy developed, indicating that monitoring serum sIL-2R may help to assessing tumor activity (at least in patients with ccRCC). Thus, our clinical observations suggested that serum sIL-2R could be used as a marker for the status of RCC, but the exact role of sIL-2R has yet to be elucidated, including how and why it is generated, the implications of an increase of sIL-2R, and how sIL-2R cooperates with other immune players. Our findings require further validation by a prospective study, preferably a larger-scale prospective controlled clinical trial. Although it is not easy to correctly assess the status of a patient with currently available methods, serial measurement of the serum level of sIL-2R may provide guidance about the current disease status. Compared with serial biopsy, a blood test is preferable as a biomarker for assessing tumor behavior and the host immune response during anticancer therapy. To confirm the clinical utility of sIL-2R with a high level of evidence, a prospective study should be performed to demonstrate that this potential biomarker can be used as the basis for clinical decisions that improve patient outcomes.

\section{Conclusions}

sIL-2R has a role in $T$ cell activation and regulation of the immune response. In patients with RCC, preoperative elevation of sIL-2R was associated with a higher serum level of PD-L1 and increased expression of phosphorylated Akt in the primary tumor, as well as a worse response to IFN-alpha and sequential VEGFtargeting therapy. Elevation of sIL-2R was also demonstrated to be an independent prognostic indicator of shorter overall survival. Furthermore, the serum level of sIL-2R largely paralleled the response to therapy over time. Our findings suggest that monitoring serum sIL-2R might facilitate assessment of the biological aggressiveness and progression of RCC, as well as helping to predict the effectiveness of treatment. Although the mechanism underlying elevation of sIL-2R in RCC is still unclear, further investigation is warranted to delineate the usefulness of sIL$2 \mathrm{R}$ for assessing disease progression and the response to therapy, as well as for designing personalized treatment.

\section{Abbreviations \\ Akt: Protein kinase B; APC: Antigen-presenting cells; ccRCC: Clear cell renal cell carcinoma; CD: Cluster of differentiation; CR: Complete response; CT: Computed tomography; ECOG: Eastern Cooperative Oncology Group; EU: European Union; Foxp3: Forkhead box P3; GLUT1: Glucose transporter protein-1; HEP: Liver; HIF: Hypoxia-inducible factor; IL: Interleukin; IL-2R: Interleukin-2 receptor; JAK: Janus kinase; KPS: Karnofsky performance status; LYM: Lymph node; MAPK: Mitogen-activated protein kinase; MMP: Matrix metalloproteinase; MRI: Magnetic resonance imaging; MSKCC: Memorial Slone-Kettering Cancer Center; mTOR: Mammalian target of rapamycin; mTORC1: mTOR-raptor complex; mTORC2: mTOR-rictor complex; OS: Overall survival time; OSS: Bone; pAkt: Phosphorylated-Akt; PD: Progressive disease; PD-1: Programmed cell death 1; PD-L1: Programmed cell death 1-ligand 1; PI3K: Phosphatidylinositol 3'kinase; PLE: Pleura; pN: Pathological Nodes; PR: Partial response; PS: Performance status; pT: Pathological Tumor; PUL: Lung; RCC: Renal cell carcinoma; RECIST: Response Evaluation Criteria in Solid Tumors; S.D.: Standard deviation; SD: Stable disease; Ser: Serine; sIL-2R: Soluble interleukin-2 receptor; STAT: Signal transducer and activator of transcription; TAM: Tumor-associated macrophages; Teff: Effector T cell; TILs: Tumor-infiltrating T lymphocytes; Treg: Regulatory T cells; USA: United States of America; VEGF: Vascular endothelial growth factor}

\section{Acknowledgements}

The authors wish to thank all patients and their families for contributing to this study, and are grateful to Hitomi Yamazaki for her excellent technical assistance.

\section{Funding}

This work was partly supported by a KAKENHI Grant (26462426) to Takao Kamai from the Japanese Science Progress Society. None of the funding bodies played a role in data collection, analysis, or interpretation of data, the writing of the manuscript, or the decision to submit the manuscript for publication.

Availability of data and materials

The datasets generated and analyzed during the current study are available from the corresponding author on reasonable request.

\section{Authors' contributions}

AN and $\mathrm{TK}^{*}$ initiated the study, participated in its design and coordination, carried out the study, performed the statistical analysis, and drafted the manuscript. AM, HA, KA carried out the study. KY participated in the design of the study and helped to draft the manuscript. We confirm that all author details in the final version are correct, that all authors have agreed to authorship and the order of authorship for this manuscript, and that all authors have the appropriate permissions and rights to the reported data. 


\section{Consent for publication}

Written informed consent was obtained from the patients for publication of this case series. A copy of the written consent is available for review by the Editor of this journal.

\section{Ethics approval and consent to participate}

This study was conducted in accordance with the Helsinki Declaration and was approved by the institutional ethical review board of Dokkyo Medical University Hospital. Each patient signed a consent form that was approved by our institutional Committee on Human Rights in Research. All samples were anonymized before analysis was performed, to guarantee the protection of privacy.

\section{Publisher's Note}

Springer Nature remains neutral with regard to jurisdictional claims in published maps and institutional affiliations.

Received: 19 October 2016 Accepted: 17 May 2017

Published online: 25 May 2017

\section{References}

1. Schreiber RD, Old LJ, Smyth MJ. Cancer immunoediting: integrating immunity's roles in cancer suppression and promotion. Science. 2011;331: 1565-70.

2. Hanahan D, Weinberg RA. Hallmarks of cancer: the next generation. Cell. 2011:144:646-74.

3. Kim $\mathrm{H}-$ J, Cantor $\mathrm{H}$. The path to reactivation of antitumor immunity and checkpoint immunotherapy. Cancer Immunol Res. 2014;2:926-36.

4. Zou W, Chen I. Inhibitory B7-family molecules in the tumour microenvironment. Nat Review Immune 2008:8:467-477.

5. Jantzer $P$, Schendel DJ. Human renal carcinoma antigen-specific CTLS, antigen-driven selection and long-term persistence in vivo. Cancer Res. 1998:58:3078-86.

6. Bromwich EJ, McArdle PA, Canna K, McMillan DC, McNicol AM, Brown M, Aitchison M. The relationship between T-lymphocyte infiltration, stage, tumour grade and survival in patients undergoing curative surgery for renal cell cancer. Br J Cancer. 2003:89:1906-8.

7. Sakaguchi S, Yamaguchi T, Nomura T, Ono M. Regulatory T cells and immune tolerance. Cell. 2008;133:775-87.

8. Cesana GC, DeRaffele G, Cohen S, Moroziewicz D, Mitcham J, Stoutenburg J, et al. Characterization of CD4+CD25+ regulatory T cells in patients treated with high-dose interleukin-2 for metastatic melanoma or renal cell carcinoma. J Clin Oncol. 2006;24:1169-77.

9. Boyman O, Sprent J. The role of interleukin-2 during homeostasis and activation of the immune system. Nat Rev Immunol. 2012;12:180-90.

10. Liao W, Lin JX, Leonard WJ. Interleukin-2 at the crossroads of effector responses, tolerance, and immunotherapy. Immunity. 2013;38:13-25.

11. Malek TR, Castro I. Interleukin-2 receptor signaling- at the interface between tolerance and immunity. Immunity. 2013;33:153-65.

12. Abe H, Kamai T. Recent advances in the treatment of metastatic renal cell carcinoma. Int J Urol. 2013;20:944-55.

13. Chang CH, Qiu J, O'Sullivan D, Buck MD, Noguchi T, Curtis JD, et al. Metabolic competition in the tumor microenvironment is a driver of cancer progression. Cell. 2015;162:1229-41.

14. Ho PC, Bihuniak JD, Macintyre AN, Staron M, Liu X, Amezquita R, et al. Phosphoenolpyruvate is a metabolic checkpoint of anti-tumor $\mathrm{T}$ cell responses. Cell. 2015;162:1217-28.

15. Furuya N, Kamai T, Shirataki H, Yanai Y, Fukuda T, Mizuno T, et al. Serum interferon alpha receptor 2 mRNA may predict efficacy of interferon alpha with/without low-dose sorafenib for metastatic clear cell renal cell carcinoma. Cancer Immunol Immunother. 2011;60:793-808.

16. Eto M, Kawano Y, Hirao Y, Mita K, Arai Y, Tsukamoto T, Hashine K, Matsubara A, Fujioka T, Kimura G, Shinohara N, Tatsugami K, Hinotsu S, Naito S, Japan RCC Trialist Collaborative Group (JRTCG) investigators. Phase II clinical trial of sorafenib plus interferon-alpha treatment for patients with metastatic renal cell carcinoma in Japan. BMC Cancer 2015;15:667.

17. Therasse P, Arbuck SG, Eisenhauer EA, Wanders J, Kaplan RS, Rubinstein L, et al. New guidelines to evaluate the response to treatment in solid tumors. European Organization for research and Treatment of Cancer, National Cancer Institute of the United States, National Cancer Institute of Canada. J Natl Cancer Inst. 2000;92:205-16.
18. Mizuno T, Kamai T, Abe H, Sakamoto S, Kitajima K, Nishihara D, et al. Clinically significant association between the maximum standardized uptake value on ${ }^{18} \mathrm{~F}-\mathrm{FDG}$ PET and expression of phosphorylated Akt and $\mathrm{S} 6$ kinase for prediction of the biological characteristics of renal cell cancer. BMC Cancer. 2015;15:114.

19. Brivio F, Lissoni P, Fumagalli L, Rovelli F, Brivio R, Vigore' L, Messina G, Tisi E. Correlation between soluble $\mathrm{IL}-2$ receptor serum levels and regulatory $T$ lymphocytes in patients with solid tumors. Int J Biol Markers 2008;23:121-122.

20. Ma WW, Jacene $H$, Song D, Vilardell F, Messersmith WA, Laheru D, et al. $\left[{ }^{18} \mathrm{~F}\right]$ Fluorodeoxyglucose positron emission tomography correlates with Akt pathway activity but is not predictive of clinical outcome during mTOR inhibitor therapy. J Clin Oncol. 2009;27:2697-704.

21. Linehan WM, Rouault TA. Molecular pathways: fumarate hydratase-deficient kidney cancer- targeting the Warburg effect in cancer. Clin Cancer Res. 2013;19:3345-52.

22. Betz C, Stracka D, Prescianotto-Baschong C, Frieden M, Demaurex N, Hall M. mTOR complex 2-Akt signaling at mitochondria-associated endoplasmic reticulum membranes (MAM) regulates mitochondrial physiology. Proc Natl Acad Sci U S A. 2013;110:12526-34.

23. Toschi A, Lee E, Gadir N, Ohh M, Foster DA. Differential dependence of hypoxia-inducible factors 1 alpha and 2 alpha on mTORC1 and mTORC2. J Biol Chem. 2008;283:34495-9.

24. Jonasch E, Corn P, Pagliaro LC, Warneke CL, Johnson MM, Tamboli $\mathrm{P}$, et al. Upfront, randomized, phase 2 trial of sorafenib versus sorafenib and lowdose interferon alfa in patients with advanced renal cell carcinoma: clinical and biomarker analysis. Cancer. 2010;116:57-65.

25. Dannull J, Su Z, Rizzieri D, Yang BK, Coleman D, Yancey D, et al. Enhancement of vaccine-mediated antitumor immunity in cancer patients after depletion of regulatory T cells. J Clin Invest. 2005;115:3623-33.

26. Kaplan RN, Riba RD, Zacharoulis S, Bramley AH, Vincent L, Costa C, et al. VEGFR1-positive haematopoietic bone marrow progenitors initiate the premetastatic niche. Nature. 2005;438:820-7.

27. Hiratsuka S, Nakamura K, Iwai S, Murakami M, Itoh T, Kijima H, et al. MMP9 induction by vascular endothelial growth factor receptor-1 is involved in lung-specific metastasis. Cancer Cell. 2002;2:289-300.

28. Yoshida N, Oda M, Kuroda Y, Katayama Y, Okikawa Y, Masunari T, et al. Clinical significance of slL-2R levels in B-cell lymphomas. PLoS One. 2013;11:e78730.

29. Gabrilovich DI, Nagaraj S. Myeloid-derived suppressor cells as regulators of the immune system. Nat Rev Immunol. 2009;9:162-74.

30. Qian BZ, Pollard JW. Macrophage diversity enhances tumor progression and metastasis. Cell. 2010;141:39-51.

31. Curran MA, Montalvo W, Yagita H, Allison JP. PD-1 and CTLA-4 combination blockade expands infiltrating $T$ cells and reduces regulatory $T$ and myeloid cells within B16 melanoma tumors. Proc Natl Acad Sci U S A. 2010;107:4275-80.

\section{Submit your next manuscript to BioMed Central and we will help you at every step:}

- We accept pre-submission inquiries

- Our selector tool helps you to find the most relevant journal

- We provide round the clock customer support

- Convenient online submission

- Thorough peer review

- Inclusion in PubMed and all major indexing services

- Maximum visibility for your research

Submit your manuscript at www.biomedcentral.com/submit
) Biomed Central 